\title{
Multiwavelength Spectrophotometric Resolution of the Micro-Equilibria of Cetirizine
}

\author{
Kin Y. TAM*广 and Luc QUÉRÉ** \\ * Sirius Analytical Instruments Ltd., Riverside, Forest Row Business Park, Forest Row, \\ East Sussex RH18 5DW, UK \\ **UCB Pharma, B-1420 Braine-l'Alleud, Belgium
}

\begin{abstract}
The acid-base properties of the antihistamine $\left(\mathrm{H}_{1}\right.$ receptor antagonist) cetirizine have been studied by using a previously developed multiwavelength spectrophotometric titration method. A new computational procedure called "Two-StepDivide-and-Conquer" (TSDC), which applied evolving factor analysis (EFA) and target factor analysis (TFA), has been derived to unravel the micro-equilibria of the triprotic zwitterionic compound from the spectral data. We have demonstrated that a single spectrophotometric titration experiment is sufficient to determine the 12 unknown microconstants and the distribution of microspecies, which are in good agreement with literature values, where available.
\end{abstract}

(Received April 16, 2001; Accepted July 23, 2001)

\section{Introduction}

The introduction of the non-sedating $\mathrm{H}_{1}$ receptor antagonists, the so-called "second-generation" antihistamines, generated a wide debate on mechanisms to explain why these compounds present little (or no) sedative effect. ${ }^{1}$ Physicochemical criteria like lipophilicity $(\log D$ or $\log P$ ), hydrogen bonding capacity $(\Delta \log P)$, protein binding and volume of distribution are

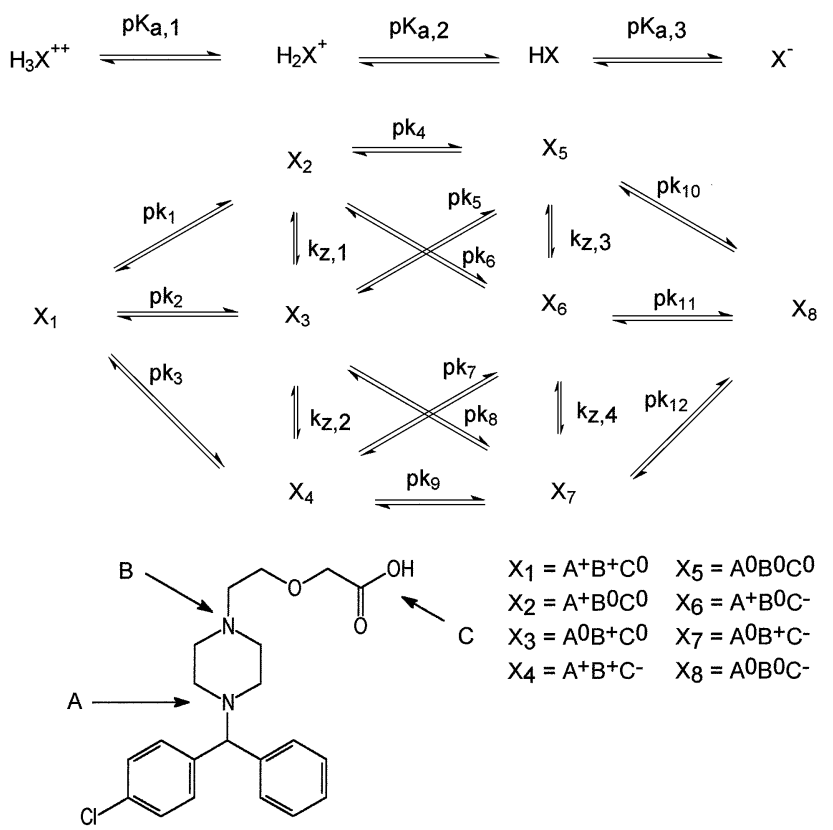

Fig. 1 Ionization scheme of cetirizine.

$\uparrow$ To whom correspondence should be addressed.

K. Y. T. present address: AstraZeneca, Mereside, Alderley Park, Macclesfield, Cheshire, SK10 4TG, UK. important issues governing not only the brain penetration but also the interactions with cytochrome $\mathrm{P} 450$ and $P$-glycoprotein. ${ }^{2}$ For compounds existing at physiological $\mathrm{pH}$ mainly as ionic forms, the extent of ionization is an important consideration since the pharmacokinetic and pharmacodynamic behaviors of various protonation forms may vary considerably.

Cetirizine belongs to this "second generation" of antihistamines with improved pharmacodynamic properties. ${ }^{3}$ It is a triprotic zwitterionic molecule, with the structure and the ionization scheme depicted in Fig. 1. The symbols $\mathrm{H}_{3} \mathrm{X}^{++}, \mathrm{H}_{2} \mathrm{X}^{+}$, $\mathrm{HX}$ and $\mathrm{X}^{-}$denote the four macrospecies, while $\mathrm{X}_{1}-\mathrm{X}_{8}$ represent the eight microspecies. Note that the macrospecies $\mathrm{H}_{3} \mathrm{X}^{++}$and $\mathrm{X}^{-}$are equivalent to the microspecies $\mathrm{X}_{1}$ and $\mathrm{X}_{8}$, respectively. Equations (1) - (12) define the twelve microconstants ( $\mathrm{p} k_{1}-\mathrm{p} k_{12}$, see also Fig. 1) and symbols $[\mathrm{H}]$ and $\left[\mathrm{X}_{n}\right](n=1, \ldots, 8)$ denote, respectively, the concentrations of the proton and microspecies. To rationalize the absorption and distribution properties of the compound, it is of great interest to define which macrospecies are present at physiological $\mathrm{pH}$ and, more importantly, to determine the relative proportion of various microspecies.

$$
\begin{aligned}
& k_{1}=\frac{[\mathrm{H}]\left[\mathrm{X}_{2}\right]}{\left[\mathrm{X}_{1}\right]} \\
& k_{2}=\frac{[\mathrm{H}]\left[\mathrm{X}_{3}\right]}{\left[\mathrm{X}_{1}\right]} \\
& k_{3}=\frac{[\mathrm{H}]\left[\mathrm{X}_{4}\right]}{\left[\mathrm{X}_{1}\right]} \\
& k_{4}=\frac{[\mathrm{H}]\left[\mathrm{X}_{5}\right]}{\left[\mathrm{X}_{2}\right]} \\
& k_{5}=\frac{[\mathrm{H}]\left[\mathrm{X}_{5}\right]}{\left[\mathrm{X}_{3}\right]} \\
& k_{6}=\frac{[\mathrm{H}]\left[\mathrm{X}_{6}\right]}{\left[\mathrm{X}_{2}\right]}
\end{aligned}
$$




$$
\begin{aligned}
& k_{7}=\frac{[\mathrm{H}]\left[\mathrm{X}_{6}\right]}{\left[\mathrm{X}_{4}\right]} \\
& k_{8}=\frac{[\mathrm{H}]\left[\mathrm{X}_{7}\right]}{\left[\mathrm{X}_{3}\right]} \\
& k_{9}=\frac{[\mathrm{H}]\left[\mathrm{X}_{7}\right]}{\left[\mathrm{X}_{4}\right]} \\
& k_{10}=\frac{[\mathrm{H}]\left[\mathrm{X}_{8}\right]}{\left[\mathrm{X}_{5}\right]} \\
& k_{11}=\frac{[\mathrm{H}]\left[\mathrm{X}_{8}\right]}{\left[\mathrm{X}_{6}\right]} \\
& k_{12}=\frac{[\mathrm{H}]\left[\mathrm{X}_{8}\right]}{\left[\mathrm{X}_{7}\right]}
\end{aligned}
$$

Recently, Testa et al. have investigated the physico-chemical properties (acid-base behavior and $\mathrm{pH}$-partitioning profiles) and pharmacokinetic parameters (plasma protein binding, brain extraction ratio and brain efflux rate) of cetirizine and have shown that the zwitterionic form $\left(\mathrm{X}_{7}\right)$ is predominant at physiological pH. ${ }^{4}$ However, Testa et al. chose to ignore the first $\mathrm{p} K_{\mathrm{a}}\left(\mathrm{p} K_{\mathrm{a}, 1}\right)$ in their calculation and the deductive approach was used to resolve the micro-equilibra. To this end, one tautomeric ratio and four microconstants were reported. ${ }^{4}$ It is noted that the deductive method makes use of the $\mathrm{p} K_{\mathrm{a}}$ value of a close derivative of the parent molecule, which contains a reduced number of ionizable groups, to derive the microconstants of the parent molecule. However, this approach assumes that the acid-base properties of the derivative and the parent are the same. This may not always be true, since the ionizable sites of these two molecules could experience different electronic and geometric environments.

In our previous study, we have developed a multiwavelength spectrophotometric " $k$ " method to investigate the microequilibria of diprotic amphoteric molecules. ${ }^{5}$ To use this method, a series of spectrophotometric titrations of the drug sample are carried out in methanol-water mixtures of different methanol contents. The molar absorption spectra of each macrospecies with the same number of bound proton(s), are first extracted by using target factor analysis. ${ }^{6,7}$ As the polarity of the solvent medium varies with methanol content, the relative proportion of the microspecies (i.e. the tautomers of the macrospecies) shifts, thus providing a handle to probe the tautomeric ratio. Based on this consideration, the molar absorption spectra of the macrospecies are deconvoluted by the $k_{z}$ method to determine the molar absorption spectra of various microspecies, the tautomeric ratio, the microconstants and the distribution of microspecies as a function of methanol content. This $k_{z}$ method has been applied successfully to deduce the tautomeric ratios and the microconstants of several diprotic amphoteric drugs ${ }^{5}$ and has been extended recently for a triprotic amphoteric compound. ${ }^{8}$

In the case of cetirizine, a triprotic zwitterionic compound with one optically inactive ioniziable group (i.e. the ionization causes negligible spectral change), all the unknown microconstants and tautomeric ratios can be determined from a single spectrophotometric titration. Specifically, one of the unknown tautomeric ratios is first determined by selective spectrophotometric monitoring of the (de)protonation of one optically active ionizable group in a suitable $\mathrm{pH}$ region, which is selected automatically by using the evolving factor analysis (EFA) method. ${ }^{9}$ With this information, all the unknown tautomeric ratios and microconstants are obtained from the full spectral data matrix by the "Target Factor Analysis" (TFA) technique as derived in this work. We called this new approach the "Two-Step-Divide-and-Conquer" (TSDC) technique. In the subsequent discussion, we will outline the EFA, TFA and the TSCD methods. Multiwavelength spectrophotometric titration data of cetirizine will be used to illustrate how the method works.

\section{Experimental}

\section{Materials}

Cetirizine (ZYRTEC ${ }^{\mathrm{TM}}$ ) was supplied by UCB Pharma (Belgium). Potassium chloride and potassium hydroxide (all AR grade) were obtained from Fisher (Loughborough, UK). Hydrochloric acid was purchased from Aldrich (Dorest, UK).

\section{Spectrophotometric titration}

All multiwavelength spectrophotometric titrations were performed using a GLpKa ${ }^{\mathrm{TM}}$ titrator in conjunction with a DPAS $^{\mathrm{TM}}$ spectrophotometric accessory (Sirius, Forest Row, UK). ${ }^{6,7}$ In brief, the optical system of the D-PAS ${ }^{\text {TM }}$ accessory consists of a pulsed deuterium lamp, a 256-element photodiode array detector and a bifurcated fiber optics dip probe. Digitized spectra were recorded and analyzed using pKaUV ${ }^{\mathrm{TM}}$ software (Sirius). The $\mathrm{pH}$ change per titrant addition was limited to about $0.1 \mathrm{pH}$ units. About 30 to 40 spectra and $\mathrm{pH}$ readings were collected from each titration. The $\mathrm{pH}$ electrode (double junction $\mathrm{Ag} / \mathrm{AgCl}$; Sentek, Braintree, UK) was calibrated titrimetrically in the $\mathrm{pH}$ range of $1.8-12.2$. All experiments were done in aqueous solution or methanol-water mixture with $0.15 \mathrm{M} \mathrm{KCl}$ under argon atmosphere at $25 \pm 0.5^{\circ} \mathrm{C}$ using standardized $0.5 \mathrm{M} \mathrm{HCl}$ or $0.5 \mathrm{M} \mathrm{KOH}$ titrants. Solutions were made up of deionized water of resistivity $>10^{14} \Omega / \mathrm{cm}$. Sample concentrations of about $10^{-5} \mathrm{M}$ were employed. Titrations were carried out in the presence of $2.5 \times 10^{-4} \mathrm{M}$ potassium dihydrogenphosphate to allow sufficient data points to be collected in the unbuffered region of the titration curve. Sample solutions were titrated from a reasonably low $\mathrm{pH}$ value $(\sim 1.8)$ to an appropriate high $\mathrm{pH}$ value ( 9.0).

\section{Results and Discussion}

In a spectrophotometric titration experiment, the spectral data obtained are a series of spectra acquired at different $\mathrm{pH}$ values, which can be expressed in the form of an absorbance matrix, $\boldsymbol{A}$, with dimension of $N_{\mathrm{s}}$ (absorption spectra) $\times N_{\mathrm{w}}$ (wavelength). According to Beer's law, $\boldsymbol{A}$ can be expressed as follows:

$$
A=C E
$$

where $\boldsymbol{C}$ and $\boldsymbol{E}$ represent, respectively, the concentration-pH profile $\left(N_{\mathrm{s}} \times N_{\mathrm{c}}\right)$ and the molar absorptivity matrix $\left(N_{\mathrm{c}} \times N_{\mathrm{w}}\right)$ with the inclusion of the optical path length. $N_{\mathrm{c}}$ is the number of independent light absorbing species (principal component) and should be less than or equal to $N_{\mathrm{s}}$ or $N_{\mathrm{w}}$, whichever is smaller. In applying the principal component analysis (PCA) procedure, the covariance matrix, $\boldsymbol{Z}$, is first calculated (Eq. (14)):

$$
Z=A^{\mathrm{T}} \boldsymbol{A}
$$

where the superscript $\mathrm{T}$ denotes a transpose operation. 
Table $1 \mathrm{p} K_{\mathrm{a}}$ values, tautomeric ratios and microconstants of cetirizine at $25^{\circ} \mathrm{C}$ and an ionic strength of 0.15

\begin{tabular}{|c|c|c|}
\hline & This work ${ }^{\mathrm{a}}$ & Testa et al..$^{4}$ \\
\hline $\mathrm{p} K_{\mathrm{a}, 1}$ & $2.12 \pm 0.04$ & $2.19 \pm 0.06$ \\
\hline $\mathrm{p} K_{\mathrm{a}, 2}$ & $2.90 \pm 0.02$ & $2.93 \pm 0.03$ \\
\hline $\mathrm{p} K_{\mathrm{a}, 3}$ & $7.98 \pm 0.02$ & $8.00 \pm 0.02$ \\
\hline $\log k_{\mathrm{z}, \mathrm{i}}^{\mathrm{b}}$ & 5.09 & 4.56 \\
\hline $\log k_{\mathrm{z}, 1}$ & 0.45 & $-^{c}$ \\
\hline $\log k_{\mathrm{z}, 2}$ & -0.67 & - \\
\hline $\log k_{\mathrm{z}, 3}$ & 1.86 & - \\
\hline $\log k_{\mathrm{z}, 4}$ & 3.23 & - \\
\hline $\mathrm{p} k_{1}$ & $2.77 \pm 0.01$ & - \\
\hline $\mathrm{p} k_{2}$ & $2.32 \pm 0.01$ & - \\
\hline $\mathrm{p} k_{3}$ & 2.99 & - \\
\hline $\mathrm{p} k_{4}$ & $7.33 \pm 0.01$ & - \\
\hline $\mathrm{p} k_{5}$ & 7.78 & 7.49 \\
\hline $\mathrm{p} k_{6}$ & $5.47 \pm 0.01$ & - \\
\hline $\mathrm{p} k_{7}$ & 5.25 & - \\
\hline $\mathrm{p} k_{8}$ & 2.70 & 2.93 \\
\hline $\mathrm{p} k_{9}$ & 2.02 & - \\
\hline $\mathrm{p} k_{10}$ & 2.89 & 3.41 \\
\hline $\mathrm{p} k_{11}$ & 4.75 & - \\
\hline $\mathrm{p} k_{12}$ & 7.98 & 8.00 \\
\hline
\end{tabular}

a. Uncertainty represents the estimated standard deviation.

b. Defined in Fig. 4 and Eq. (22)

c. Not available.

Diagonalization of the covariance matrix gives the eigenvector $\boldsymbol{Q}$ and eigenvalue $\boldsymbol{\lambda}$ (Eq. (15)):

$$
Z Q=Q \lambda
$$

It should be remarked that only those components with large eigenvalues contribute significantly to the observed data, while others are regarded as noise and can be discarded. In the present study, residual standard deviation, ${ }^{11}$ IND function, ${ }^{10,11}$ eigenvalue ratio $^{12}$ and reduced eigenvalue ratio ${ }^{13,14}$ are adopted to identify the number of principal components $N_{\mathrm{c}}$ that are important in describing the absorbance matrix.

The eigenvector and eigenvalue matrices with selected principal components are symbolized by $\boldsymbol{Q}_{\mathrm{r}}$ and $\boldsymbol{\lambda}_{\mathrm{r}}$, respectively. From these, we can compute an abstract solution for the molar absorptivity matrix $\left(\boldsymbol{E}_{\text {abs }}\right)$ and concentration-pH profile matrix $\left(\boldsymbol{C}_{\mathrm{abs}}\right)$ by using the following equations (Eqs. (16) and (17)):

$$
\begin{aligned}
& \boldsymbol{E}_{\mathrm{abs}}=\boldsymbol{Q}_{\mathrm{r}}^{\mathrm{T}} \\
& \boldsymbol{C}_{\mathrm{abs}}=\boldsymbol{A} \boldsymbol{Q}_{\mathrm{r}}
\end{aligned}
$$

The abstract solution can be rotated to the solution with physical significance, $\boldsymbol{E}_{\mathrm{p}}$ and $\boldsymbol{C}_{\mathrm{p}}$, via a transformation matrix $\boldsymbol{T}$ as defined below: ${ }^{11,15,16}$

$$
\begin{aligned}
& \boldsymbol{T}=\boldsymbol{\lambda}_{\mathrm{r}}^{-1} \boldsymbol{C}_{\mathrm{abs}}^{\mathrm{T}} \boldsymbol{C}_{\mathrm{t}} \\
& \boldsymbol{A} \approx \boldsymbol{C}_{\mathrm{abs}} \boldsymbol{T} \boldsymbol{T}^{-1} \boldsymbol{E}_{\mathrm{abs}} \\
& \approx \boldsymbol{C}_{\mathrm{p}} \boldsymbol{E}_{\mathrm{p}}
\end{aligned}
$$

where the superscript -1 denotes an inverse operation. The test matrix $\boldsymbol{C}_{\mathrm{t}}$ in Eq. (18) contains the concentration-pH profiles of the principal components, which are generated by the equations as derived below. In this study, the proton concentration is

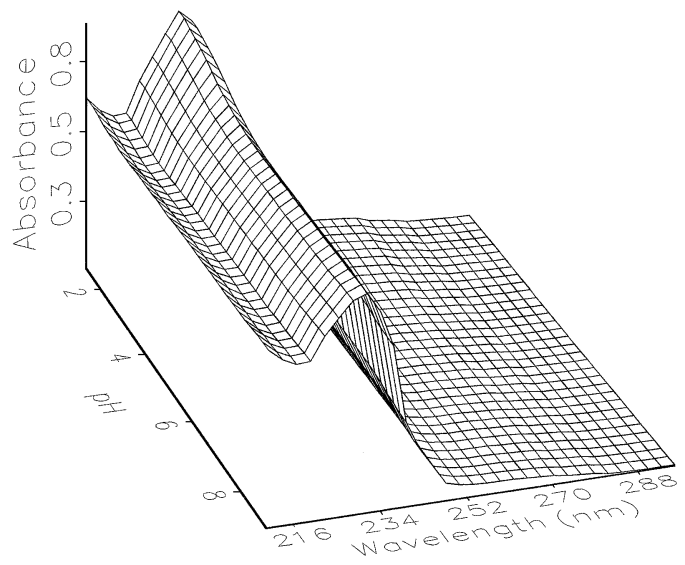

Fig. 2 Absorption spectra of cetirizine.

related to the operational $\mathrm{pH}$ reading by a multi-parametric equation. ${ }^{17}$

The SPOIL function as proposed by Malinowski ${ }^{11,16}$ is utilized to determine whether a test matrix is acceptable or not. The SPOIL function reflects the deviation between $\boldsymbol{C}_{\mathrm{t}}$ and $\boldsymbol{C}_{\mathrm{p}}$. In this context, a test matrix that minimizes the SPOIL function with a value not greater than 3.0 is considered as the solution for the target transformation procedure. ${ }^{11,16}$ In other words, the calculation seeks to minimize the deviation between $\boldsymbol{C}_{\mathrm{t}}$ and $\boldsymbol{C}_{\mathrm{p}}$ to give a best fit between theory and experiment. For a particular $\boldsymbol{A}$, the SPOIL function depends only on $\boldsymbol{C}_{\mathrm{t}}$, which in turn is a function of the sought $\mathrm{p} K_{\mathrm{a}} \mathrm{s}$ or microconstants. Here, we define a cost function, $\Phi$

$$
\Phi=\xi+\zeta+\sum_{i=1}^{N \mathrm{c}}\left(\mathrm{SPOIL}_{i}\right)^{2}
$$

where the symbol $\xi$ represents a penalty function for any negative element in the $\boldsymbol{E}_{\mathrm{p}}$ matrix. $\boldsymbol{\zeta}$ denotes a penalty function for the $\mathrm{p} K_{\mathrm{a}}$ or microconstant values which is activated if the sought values diverge from certain specified feasible ranges. The TFA computation renders to a constrained optimization of the adjustable parameters for a global minimum of $\Phi$. The SIMPLEX method can be used for this purpose. ${ }^{18}$

Table 1 lists the $\mathrm{p} K_{\mathrm{a}}$ values of cetirizine as determined by $\mathrm{pH}$ metric titration, which are in excellent agreement with literature values obtained using the same method. The details of the $\mathrm{pH}$ metric technique for $\mathrm{p} K_{\mathrm{a}}$ determination of cetirizine have been reported $^{4}$ and are not repeated here. The absorption spectra of cetirizine obtained from a D-PAS titration are depicted in Fig. 2. Principal component analysis on this data matrix revealed only three independent light absorbing species, suggesting that two of the three ionizable groups, namely the two nitrogen moieties (groups A and B in Fig. 1), may generate detectable spectral changes upon (de)protonation. It is plausible that the $-\mathrm{COOH}$ moiety (group $\mathrm{C}$ in Fig. 1) is optically inactive since it is relatively far away (five $\sigma$ bonds) from the chromophore. In the subsequent discussion, we will use the "Two-Step-Divide-andConquer" (TSDC) strategy to resolve all the unknown microconstants of cetirizine from a single experiment.

\section{Step 1: Selective monitoring of the ionization of group B}

In the presence of an optically inactive group (C), the ionization of group B could be selectively monitored using a suitably selected $\mathrm{pH}$ region, in which the spectral shifts contributed by group A are negligible. Within this favorable $\mathrm{pH}$ region, we can simplify the calculation to allow one tautomeric 


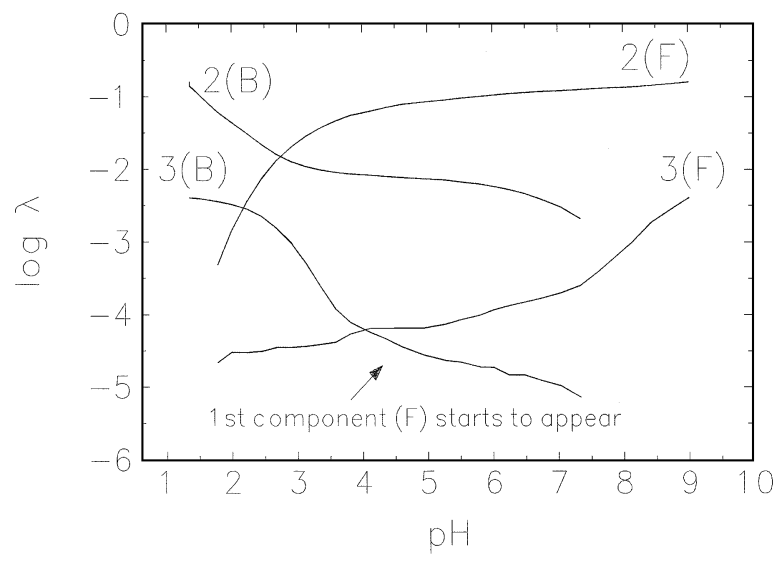

Fig. 3 Logarithmic plot of the evolution of the eigenvalues for cetirizine. $2(\mathrm{~F})$ and $3(\mathrm{~F})$ are the second and third eigenvalues in the forward direction, whereas 2(B) and 3(B) are the second and third eigenvalues in the backward direction.

ratio to be determined unambiguously.

To define a suitable $\mathrm{pH}$ region for this purpose, we apply evolving factor analysis (EFA) to the spectral data shown in Fig. 2. In brief, EFA is a model-free method, which is based on repetitive eigenvalue analysis (Eqs. (14) and (15)) of a set of data matrices generated from the titration process. Eigenvalue analyses are performed on a series of matrices constructed by successively adding spectra to the previous matrix during the titration. When a new absorbing species appears, an eigenvalue evolves from the pool of error eigenvalues and increases its contribution to the full data matrix. This is called forward evolving factor analysis. On the other hand, backward evolving factor analysis is carried out by starting the eigenvalue analysis with the last few spectra and including spectra in the reverse order of the data collection. A plot of the eigenvalues obtained from the eigenvalue analyses provides valuable information. Note that the area under the $i$ th forward eigenvalue curve and the $(n+1-i)$ th backward eigenvalue curve maps out qualitatively the concentration profile of the $i$ th species. Figure 3 shows the eigenvalues as a function of $\mathrm{pH}$ obtained from the forward and reverse analyses. It can be seen that the 3rd component (backward), which corresponds to the 1st component (forward), starts to appear at $\mathrm{pH}$ below 4.5. This suggests that the spectral contribution from this component is negligible above $\mathrm{pH}$ 4.5. Consequently, the TFA method ${ }^{6,7}$ is applied to the spectral data collected between $\mathrm{pH} 4.5$ - 9, with the $\boldsymbol{C}_{\mathrm{t}}$ matrix generated as below.

As shown in Table 1, $\mathrm{p} K_{\mathrm{a}, 1}$ of cetirizine is 2.12 , which is more than $2 \mathrm{pH}$ units below the minimum $\mathrm{pH}$ value under consideration. It is therefore reasonable to ignore $\mathrm{p} K_{\mathrm{a}, 1}$ and the ionization of group A. Based on these assumptions, a simplified ionization model of cetirizine is established in Fig. 4. Note that we designate the microconstants differently from the system used in Fig. 1. Since these parameters are derived from a simplified model, it is expected that the values may not be the same as the corresponding microconstants as defined in Fig 1. Nevertheless, the tautomeric ratio, $k_{\mathrm{z}, \mathrm{i}}$, remains the same, regardless of the simplified model (Fig. 4) or the full model (Fig. 1). This notion can be expressed as follows:

$$
k_{\mathrm{z}, \mathrm{i}}=\frac{k_{8}{ }^{\prime}}{k_{5}{ }^{\prime}}=\frac{k_{8}}{k_{5}}
$$

To generate the $\boldsymbol{C}_{\mathrm{t}}$ matrix, the distribution of microspecies is

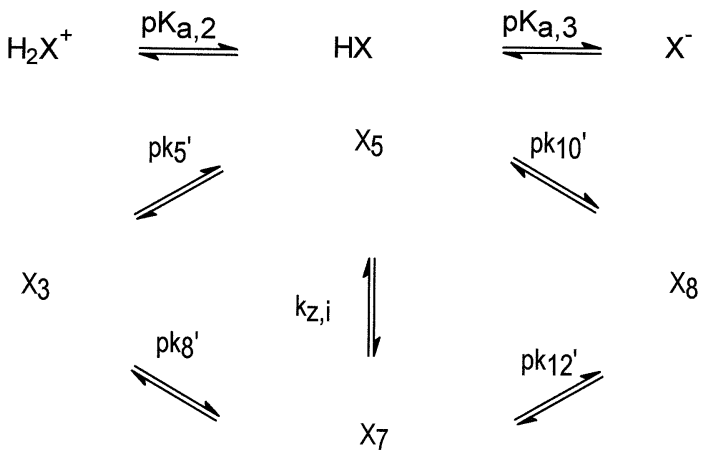

Fig. 4 Simplified ionization scheme of cetirizine above $\mathrm{pH} 4.5$, with the microspecies defined in Fig. 1.

first calculated by solving the following matrix equation: ${ }^{5}$

$$
\left[\begin{array}{l}
Y \\
0 \\
0 \\
0
\end{array}\right]=\left[\begin{array}{cccc}
1 & 1 & 1 & 1 \\
k_{8}^{\prime} & -[\mathrm{H}] & 0 & 0 \\
0 & k_{12}^{\prime} & -[\mathrm{H}] & 0 \\
0 & 0 & -[\mathrm{H}] & k_{10}{ }^{\prime}
\end{array}\right]\left[\begin{array}{l}
{\left[\mathrm{X}_{3}\right]} \\
{\left[\mathrm{X}_{7}\right]} \\
{\left[\mathrm{X}_{8}\right]} \\
{\left[\mathrm{X}_{5}\right]}
\end{array}\right]
$$

in which $Y$ denotes the initial concentration of the sample used. The symbols $k_{12}{ }^{\prime}$ and $k_{10}{ }^{\prime}$ can be expressed in terms of $k_{8}{ }^{\prime}, K_{\mathrm{a}, 2}$ and $K_{\mathrm{a}, 3}$ by using the following Eqs.:

$$
\begin{aligned}
& k_{12}^{\prime}=\frac{K_{\mathrm{a}, 2} K_{\mathrm{a}, 3}}{k_{8}^{\prime}} \\
& k_{10^{\prime}}=\frac{K_{\mathrm{a}, 2} K_{\mathrm{a}, 3}}{k_{5}^{\prime}}=\frac{K_{\mathrm{a}, 2} K_{\mathrm{a}, 3}}{K_{\mathrm{a}, 2}-k_{8}{ }^{\prime}}
\end{aligned}
$$

Then, the principal components to form the $\boldsymbol{C}_{\mathrm{t}}$ matrix are established as below:

$$
\boldsymbol{C}_{\mathrm{t}}^{\mathrm{T}}=\left[\begin{array}{l}
{[\mathrm{X} 3]+[\mathrm{X} 7]} \\
{[\mathrm{X} 5]+[\mathrm{X} 8]}
\end{array}\right]
$$

where the first row denotes the species with group B protonated and the second row indicates the species with group B uncharged. From Eqs. (18) and (21), the first step TFA computation renders an optimization of $k_{8}{ }^{\prime}$. It was found that the calculations successfully converged with each SPOIL value <1.0. This optimized parameter was consistent with the corresponding microconstant, as determined using the full model (within $0.2 \mathrm{pH}$ units). As shown in Table 1 , the $\log k_{\mathrm{z}, \mathrm{t}}$ value (as calculated from Eqs. (22) and (25)) agrees well with a literature value of 4.56 as determined by a deductive method. ${ }^{4}$

\section{Step 2: Complete resolution of the micro-equilibria with $k_{z, i}$ as a} constraint

Next, we turn to the determination of the 12 unknown microconstants. The full data matrix as shown in Fig. 2 is subjected to the TFA treatment. As mentioned before, only three independent light absorbing species were identified by the principal component analysis. Therefore, the $\boldsymbol{C}_{\mathrm{t}}$ matrix consists of only three principal components, which may be assigned as follows:

$$
\boldsymbol{C}_{\mathrm{t}}^{\mathrm{T}}=\left[\begin{array}{r}
{\left[\mathrm{X}_{1}\right]+\left[\mathrm{X}_{2}\right]+\left[\mathrm{X}_{4}\right]+\left[\mathrm{X}_{6}\right]} \\
{\left[\mathrm{X}_{3}\right]+\left[\mathrm{X}_{7}\right]} \\
{\left[\mathrm{X}_{5}\right]+\left[\mathrm{X}_{8}\right]}
\end{array}\right]
$$


where the first row represents the species with group A protonated, the second row denotes the species with group $\mathrm{A}$ uncharged and group B protonated and the third row indicates the species with group A and group B uncharged. To generate the $\boldsymbol{C}_{\mathrm{t}}$ matrix, it is required to solve the distribution of the eight microspecies as a function of pH. Based on Eqs. (1), (2), (4), (6), (7), (8), and (10), the distribution of microspecies can be calculated by solving the following matrix equation:

$$
\left[\begin{array}{l}
Y \\
0 \\
0 \\
0 \\
0 \\
0 \\
0 \\
0
\end{array}\right]=\left[\begin{array}{cccccccc}
1 & 1 & 1 & 1 & 1 & 1 & 1 & 1 \\
k_{1} & -[\mathrm{H}] & 0 & 0 & 0 & 0 & 0 & 0 \\
k 2 & 0 & -[\mathrm{H}] & 0 & 0 & 0 & 0 & 0 \\
0 & k_{4} & 0 & 0 & -[\mathrm{H}] & 0 & 0 & 0 \\
0 & k 6 & 0 & 0 & 0 & -[\mathrm{H}] & 0 & 0 \\
0 & 0 & 0 & k 7 & 0 & -[\mathrm{H}] & 0 & 0 \\
0 & 0 & k 8 & 0 & 0 & 0 & -[\mathrm{H}] & 0 \\
0 & 0 & 0 & 0 & k_{10} & 0 & 0 & -[\mathrm{H}]
\end{array}\right]\left[\begin{array}{l}
{\left[\mathrm{X}_{1}\right]} \\
{\left[\mathrm{X}_{2}\right]} \\
{\left[\mathrm{X}_{3}\right]} \\
{\left[\mathrm{X}_{4}\right]} \\
{\left[\mathrm{X}_{5}\right]} \\
{\left[\mathrm{X}_{6}\right]} \\
{\left[\mathrm{X}_{7}\right]} \\
{\left[\mathrm{X}_{8}\right]}
\end{array}\right]
$$

in which $Y$ denotes the initial concentration of the sample used.

The microconstants, $k_{3}, k_{5}, k_{7}, k_{8}, k_{9}, k_{10}, k_{11}$ and $k_{12}$ can be expressed in terms of $k_{1}, k_{2}, k_{4}, k_{6}$ and the known $\mathrm{p} K_{\mathrm{a}}$ values by using the following equations:

$$
\begin{aligned}
& k_{3}=K_{\mathrm{a}, 1}-k_{1}-k_{2} \\
& k_{5}=\frac{k_{1} k_{4}}{k_{2}} \\
& k_{7}=\frac{k_{1} k_{6}}{k_{3}} \\
& k_{8}=\frac{K_{\mathrm{a}, 1} K_{\mathrm{a}, 2}-k_{1} k_{4}-k_{1} k_{6}}{k_{2}} \\
& k_{9}=\frac{k_{2} k_{8}}{k_{3}} \\
& k_{10}=\frac{K_{\mathrm{a}, 1} K_{\mathrm{a}, 2} K_{\mathrm{a}, 3}}{k_{1} k_{4}} \\
& k_{11}=\frac{K_{\mathrm{a}, 1} K_{\mathrm{a}, 2} K_{\mathrm{a}, 3}}{k_{1} k_{6}} \\
& k_{12}=\frac{K_{\mathrm{a}, 1} K_{\mathrm{a}, 2} K_{\mathrm{a}, 3}}{k_{2} k_{8}}
\end{aligned}
$$

From Eqs. (28) - (36), it can be seen that the $\boldsymbol{C}_{\mathrm{t}}$ matrix is a function of $k_{1}, k_{2}, k_{4}$ and $k_{6}$. These are treated as adjustable parameters in the second step TFA computation to minimize the modified cost function, $\Theta$, as defined below:

$$
\Theta=\Phi+P
$$

where the symbol $P$ represents a penalty function for the $k_{\mathrm{z}, \mathrm{i}}$ value, which is activated if it diverges from the $k_{z, \mathrm{i}}$ value determined in Step 1 (see Eq. (22)). The variable $\Phi$ is evaluated by Eq. (21). It was found that the calculation successfully converged with each SPOIL value $<1.0$ and all constraints satisfied. Table 1 lists the microconstants and tautomeric ratios of cetirizine, which are consistent with literature values where available. ${ }^{4}$ It is worth noting that $\mathrm{p} k_{3}, \mathrm{p} k_{8}$ and $\mathrm{p} k_{10}$ are lower than the acid dissociation constant of carboxylic acid $(\sim 4)$. This is probably because cetirizine is a rather flexible molecule, in which group $\mathrm{C}$ could form an intramolecular hydrogen bond with group $\mathrm{B},{ }^{4}$ so enhancing the acidity of the former. Figures
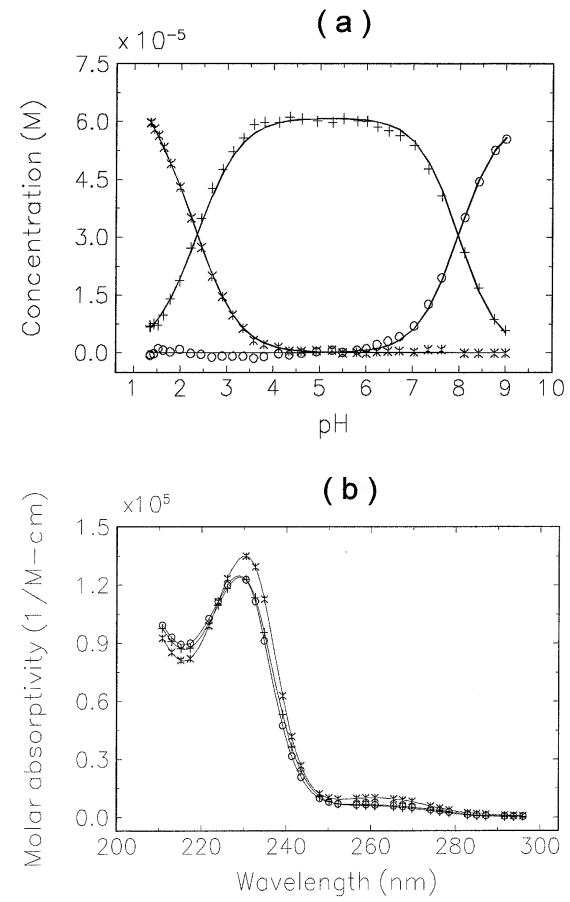

Fig. 5 (a) Distribution of species as a function of $\mathrm{pH}$ for cetirizine. The solid lines represent the $\boldsymbol{C}_{\mathrm{t}}$ matrix and the symbols represent the $\boldsymbol{C}_{\mathrm{p}}$ matrix $\left(*\left(\left[\mathrm{X}_{1}\right]+\left[\mathrm{X}_{2}\right]+\left[\mathrm{X}_{4}\right]+\left[\mathrm{X}_{6}\right]\right),+\left(\left[\mathrm{X}_{3}\right]+\left[\mathrm{X}_{7}\right]\right), o\left(\left[\mathrm{X}_{5}\right]+\right.\right.$ $\left.\left.\left[\mathrm{X}_{8}\right]\right)\right)$. (b) Molar absorption coefficients of cetirizine, in which the symbols represent the $\boldsymbol{E}_{\mathrm{p}}$ matrix.

5(a) and (b) show, respectively, the distribution of species and the resolved molar absorption spectra of cetirizine. It can be seen that the shape of the distribution of species plot (Fig. 5(a)) is qualitatively in line with the logarithmic eigenvalue plot as derived in Step 1 (Fig. 3), suggesting that the EFA method will be useful to gain mechanistic insights, without prior knowledge of the ionization process. Figure 6 depicts the distribution of microspecies for cetirizine as generated using Eq. (28) and the microconstants reported in Table 1. It can be seen that the zwitterionic species, $\mathrm{X}_{7}$, is the predominant species between $\mathrm{pH}$ 3 to 8 , which is in good agreement with the literature. ${ }^{4}$

\section{Concluding Remarks}

The micro-equilibria of cetirizine, a triprotic zwitterionic histamine $\mathrm{H}_{1}$ receptor antagonist, have been investigated by a multiwavelength spectrophotometric titration technique. The unknown microconstants were successfully determined by using the TSDC technique developed in this study. We first applied the EFA technique to map out the $\mathrm{pH}$ region in which only one ionizable site showed $\mathrm{pH}$-dependent spectral shifts. From these, one of the unknown tautomeric ratios was determined and used as a constraint for the subsequent TFA calculation to determine other unknown microconstants. To this end, we have shown that a single spectrophotometric titration experiment was sufficient to determine the 12 unknown microconstants and the distribution of microspecies, which are in good agreement with the literature results, where available. It is noted that cetirizine exists predominately as a zwitterion $\left(\mathrm{X}_{7}\right.$; see Fig. 6) over more than $4 \mathrm{pH}$ units. This is mainly due to the intramolecular interaction between groups $\mathrm{B}$ and $\mathrm{C}$, giving additional stabilization for this zwitterionic form. ${ }^{4}$ It is plausible that this intramolecular charge neutralization may enhance the 


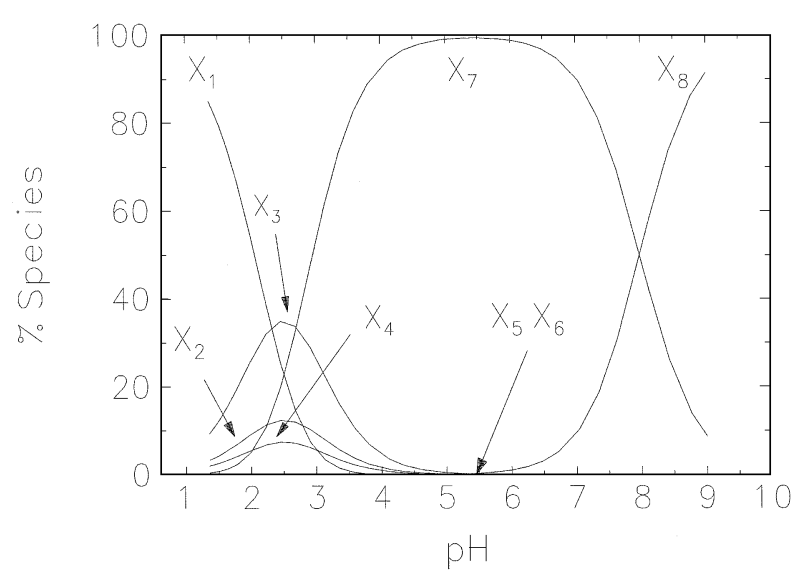

Fig. 6 Distribution of microspecies for cetirizine generated using Eq. (28) and the parameters listed in Table 1

pharmacokinetic properties of cetirizine. It would be interesting to compare the micro-equilibria of cetirizine with another "rigid" zwitterionic antihistamine of lower bioavailability, like fexofenadine, to rationalize the interplay between intramoleculer interaction and molecular conformation. Further work on this aspect is being carried out in our laboratories and results will be reported in due course.

\section{Acknowledgements}

We thank John Comer (Sirius) for various helpful comments.

\section{References}

1. H. Timmerman, Clin. Exp. Allergy, 1999, 29, 13.

2. J. P. Rihoux and J. M. Nicolas, Inflamm. Res., 1999, 48, S71.

3. J. P. Rihoux, Ann. Allergy Asthma Immunol., 1999, 83, 489.

4. A. Pagliara, B. Testa, P. A. Carrupt, P. Jolliet, C. Morin, D. Morin, S. Uroem, J. P. Tillement, and J. P. Rihoux, J. Med. Chem., 1998, 41, 853.

5. K. Takács-Novák and K. Y. Tam, J. Pharm. Biomed. Anal., 2000, 21, 1171.

6. R. I. Allen, K. J. Box, J. E. A. Comer, C. Peake, and K. Y. Tam, J. Pharm. Biomed. Anal., 1998, 17, 699.

7. K. Y. Tam and K. Takács-Novák, Pharm. Res., 1999, 16 , 374.

8. K. Y. Tam, Mikrochim. Acta, 2001, 136, 91.

9. L. Norgaard, Anal. Chim. Acta, 1991, 255, 143

10. E. R. Malinowski, Anal. Chem., 1977, 49, 612.

11. E. R. Malinowski, "Factor Analysis in Chemistry", 1991, Wiley, New York.

12. H. B. Woodruff, P. C. Tway, and L. J. C. Love, Anal. Chem., 1981, 53, 81.

13. P. J. Gemperline and J. C. Hamilton, in "ComputerEnhanced Analytical Spectroscopy, vol. 2”, ed. H. L. C. Meuzelaar, 1990, Plenum, New York, 27.

14. E. R. Malinowski, J. Chemom., 1987, 1, 33.

15. M. D'Ambiose and B. Lagarde, Comput. Chem., 1989, 13, 39.

16. M. McCue and E. R. Malinowski, Appl. Spectrosc., 1983, 37, 463.

17. A. Avdeef and J. J. Bucher, Anal. Chem., 1978, 50, 2137.

18. J. A. Nelder and R. Mead, Comput. J., 1965, 7, 308. 